\title{
Resultados materno fetais de complicações na gestação com câncer de ovário
}

\author{
Maternal fetal outcomes of pregnancy complications with ovarian cancer \\ Resultados maternos fetales de las complicaciones del embarazo com cáncer de ovario
}

Vitoria Vilas Boas da Silva Bomfim ORCID: https://orcid.org/0000-0003-4897-0279

Centro Universitário Jorge Amado, Brasil E-mail: pesquisaclinica9@gmail.com Andressa Fernandes da Silva

ORCID: https://orcid.org/0000-0002-1942-0860 Instituto Nacional de Cardiologia, Brasil E-mail: andressafdurso@gmail.com

Andrezza Eduarda Borba Gomes ORCID: https://orcid.org/0000-0003-1263-0192 Centro Universitário São Miguel, Brasil E-mail: andrezzaborba@gmail.com

Kelly Alencar de Souza ORCID: https://orcid.org/0000-0001-7372-3566 Faculdade São Francisco da Paraíba, Brasil E-mail: kellyalencar1@hotmail.com

Maria Eduarda Lopes de Macedo Bezerra ORCID: https://orcid.org/0000-0001-6741-3190 Faculdade Estácio do Rio Grande do Norte, Brasil E-mail: eduardalopes022@gmail.com

Yesly Marinho da Rocha Barreto ORCID: https://orcid.org/0000-0002-5610-1420 Centro Universitário Brasileiro, Brasil E-mail: marinhoyesly@gmail.com

Sirlene Aparecida de Oliveira ORCID: https://orcid.org/0000-0003-2352-7983 Faculdade de Ensino Superior de Cacoal, Brasil

E-mail: sirleneaparecidadeoliveira1988@gmail.com Thiemmy de Souza Almeida Guedes ORCID: https://orcid.org/0000-0003-2261-0320 Faculdade de Venda Nova do Imigrante, Brasil E-mail: thiemmyalmeida@gmail.com

Livia Maria Tavares Miranda ORCID: https://orcid.org/0000-0002-7638-6208

Faculdade Vernda Nova do Imigrante, Brasil E-mail: livia.miranda12@gmail.com

Walisson da Silva Vieira

ORCID: https://orcid.org/0000-0002-3291-2069 Faculdade Estácio do Rio Grande do Norte, Brasil

E-mail: walissonvieira.2010@gmail.com

Jônatas Lucas Marcelino da Silva ORCID: https://orcid.org/0000-0002-4063-2104

Universidade Federal de Pernambuco, Brasil E-mail: jonatas.silva@ufpe.br

Samara Dantas de Medeiros Diniz ORCID: https://orcid.org/0000-0001-9418-0185 Faculdade Estácio do Rio Grande do Norte, Brasil E-mail:samaradantas1998@gmail.com

Angelica Ribeiro do Nascimento Oliveira ORCID: https://orcid.org/0000-0002-7240-2004 Centro Universitário Maurício de Nassau, Brasil E-mail: angelicaribeiro.ar19@gmail.com

Andreia Tomaz da Silva

ORCID: https://orcid.org/0000-0002-5262-2923 Faculdade Integrada Diferencial, Brasil E-mail: andreia_tomazz@yahoo.com.br

William França dos Santos

ORCID: https://orcid.org/0000-0001-5750-7740

Universidade Federal de Pernambuco, Brasil E-mail: williamfds1997@gmail.com 


\begin{abstract}
Resumo
O câncer de ovário continua sendo o tipo mais mortal de ginecologia malignidade nos Estados Unidos. enquanto houver vários subtipos histológicos de câncer de ovário, os tipos de células epiteliais são a variante mais comum e, portanto, contribui mais para a morbidade e mortalidade atribuídas a malignidade ovariana. Portanto, realizamos uma revisão sistemática da literatura para delinear as características e resultados da EOC durante gravidez. Esta revisão foi conduzida com base na diretriz MOOSE para revisões sistemáticas usando motores de busca públicos PubMed e MEDLINE. As palavras-chave de entrada usadas para a pesquisa foram " gravidez " e " câncer de ovário ". A busca foi limitada a artigos publicado em inglês entre janeiro de 1955 e novembro de 2013. As principais conclusões da nossa revisão da literatura são que as mães os resultados da gravidez complicada por EOC são altamente contingentes no estágio do diagnóstico e tipo histológico. A maioria de gestações geridas com expectativa resultaram em sucesso neonatal resultados. Eventos adversos graves durante a gravidez foram menos frequentes do que foi observado em outros tipos histológicos de tumor ovariano.
\end{abstract}

Palavras-chave: Gravidez; Câncer de ovário; Complicações.

\begin{abstract}
Ovarian cancer remains the deadliest type of gynecology malignancy in the United States. while there are several histological subtypes of ovarian cancer, epithelial cell types are the most common variant and therefore contribute most to the morbidity and mortality attributed to ovarian malignancy. Therefore, we performed a systematic review of the literature to delineate the characteristics and outcomes of EOC during pregnancy. This review was conducted based on the MOOSE guideline for systematic reviews using public search engines PubMed and MEDLINE. The input keywords used for the search were "pregnancy" and "ovarian cancer". The search was limited to articles published in English between January 1955 and November 2013. The main conclusions of our review of the literature are that mothers outcomes of pregnancy complicated by EOC are highly contingent on the stage of diagnosis and histological type. The majority of expectantly managed pregnancies resulted in successful neonatal outcomes. Serious adverse events during pregnancy were less frequent than observed in other histological types of ovarian tumor.
\end{abstract}

Keywords: Pregnancy; Ovary cancer; Complications.

\title{
Resumen
}

El cáncer de ovario sigue siendo el tipo de malignidad ginecológica más mortal en los Estados Unidos. Si bien existen varios subtipos histológicos de cáncer de ovario, los tipos de células epiteliales son la variante más común y, por lo tanto, contribuyen más a la morbilidad y mortalidad atribuidas a la neoplasia maligna de ovario. Por lo tanto, realizamos una revisión sistemática de la literatura para delinear las características y los resultados de la COE durante el embarazo. Esta revisión se realizó sobre la base de la guía MOOSE para revisiones sistemáticas utilizando motores de búsqueda públicos PubMed y MEDLINE. Las palabras clave de entrada utilizadas para la búsqueda fueron "embarazo" y "cáncer de ovario". La búsqueda se limitó a artículos publicados en inglés entre enero de 1955 y noviembre de 2013. Las principales conclusiones de nuestra revisión de la literatura son que los resultados maternos del embarazo complicado por EOC dependen en gran medida de la etapa del diagnóstico y del tipo histológico. La mayoría de los embarazos manejados de manera expectante dieron como resultado resultados neonatales exitosos. Los eventos adversos graves durante el embarazo fueron menos frecuentes que los observados en otros tipos histológicos de tumores de ovario.

Palabras clave: El embarazo; Cáncer de ovario; Complicaciones.

\section{Introdução}

O câncer de ovário continua sendo o tipo mais mortal de ginecologia malignidade nos Estados Unidos (Morgan et al., 2013; Siegel et al., 2014). Enquanto houver vários subtipos histológicos de câncer de ovário, os tipos de células epiteliais são a variante mais comum e, portanto, contribui mais para a morbidade e mortalidade atribuídas a malignidade ovariana (Quirk et al., 1999).

Os tumores epiteliais serosos têm a maior incidência, observou a aproximadamente $70 \%$ dos tumores de origem epitelial, enquanto outros subtipos compreendem os casos restantes, a maioria comumente mucinosas, endometrióides e células claras (Irving et al., 2006; McCuluggage et al., 2011).

Demograficamente, as mulheres brancas têm a maior incidência de doenças epiteliais cânceres de ovário (EOCs), com aproximadamente 16,5 casos por 100.000 mulheres por ano sendo anotadas por meio de dados coletados em um banco de dados populacional. Embora quase metade dos casos de câncer de ovário sejam relatado em mulheres com mais de 60 anos de idade, uma significativa porcentagem de casos são pobres em mulheres em idade reprodutiva (Quirk et al., 1999). 
A EOC geralmente se apresenta de maneira insidiosa. As manifestações são tipicamente observada apenas após a presença de doença avançada e inclui pélvica ou dor abdominal, distensão abdominal, diminuição do apetite ou saciedade precoce e mudanças nos hábitos intestinais ou da bexiga (Marcus et al., 2014; SEER, 2013; Olson et al., 2001). O câncer durante a gravidez é uma entidade clínica rara, afetando aproximadamente 0,1-0,2\% das mulheres grávidas (Antonelli et al.,1996; Moran et al., 2007).

Cancro do ovário representa o quinto câncer mais comum administrado durante gravidez. Apesar da prevalência relativamente baixa de EOC em mulheres em idade reprodutiva, os tipos de células epiteliais compreendem aproximadamente 25-50\% das neoplasias ovarianas formadas em gravidez. A gravidez é caracterizada por múltiplos mudanças fisiológicas, incluindo excesso de sexo endógeno e hormônios de crescimento, que podem afetar o curso clínico de uma malignidade de maneiras imprevisíveis. O manejo do câncer na gravidez é complicado por três separados, mas interagindo entidades que devem ser gerenciadas simultaneamente: a mãe, feto e malignidade (Smith et al., 2001; Grimm et al., 2014; Kwon et al., 2010).

EOC é uma complicação rara da gravidez; no entanto, como tem o potencial para ser uma doença maligna extremamente agressiva, é imperativo que seu manejo ideal durante a gravidez é definido. Ainda restam dúvidas sobre como alterações fisiológicas durante a gravidez complicaram o curso clínico e metodologias de tratamento para EOC. Além disso, o impacto da EOC sobre o resultado fetal ainda não foi completamente compreendido. Portanto, realizamos uma revisão sistemática da literatura para delinear as características e resultados da EOC durante gravidez.

\section{Metodologia}

Esta revisão foi conduzida com base na diretriz MOOSE para revisões sistemáticas usando motores de busca públicos PubMed e MEDLINE. As palavras-chave de entrada usadas para a pesquisa foram " gravidez " e " câncer de ovário ". A busca foi limitada a artigos publicado em inglês entre janeiro de 1955 e novembro de 2013 (Stroup et al.,2000; Estrela, 2018).

Os critérios de inclusão foram relatos de casos ou séries de casos que tiveram uma descrição adequada para (i) dados demográficos clínicos, (ii) gravidez resultados, (iii) característica do tumor, (iv) tipo de intervenção e tratamento e (v) resultado de sobrevivência materna. Referências para cada artigo avaliado também foram revisados, e os correspondentes artigos que atenderam aos critérios de inclusão foram incluídos no estudo. Os artigos excluídos incluíam revisões e relatos de caso com descrição abaixo do ideal, ou se o diagnóstico de câncer de ovário foi feito antes da gravidez ou durante o pós-parto período.

Entre os artigos elegíveis, as seguintes variáveis foram abstraídas da descrição do caso: (i) dados demográficos clínicos incluindo ano e país de publicação, idade no diagnóstico, paridade, gestacional idade no diagnóstico de câncer de ovário e sintomas de apresentação; (ii) resultados da gravidez, incluindo a idade gestacional no parto, via de parto, peso ao nascer, escores de Apgar e neonatal complicações; (iii) características do tumor, incluindo histológicas subtipo, lateralidade do tumor e tamanho / peso e câncer de ovário etapa; (iv) tipo de intervenção e tratamento para câncer de ovário incluindo cirurgia de tumor em idade gestacional, tipo e tempo de quimioterapia e tipo e horário de radioterapia; e (v) resultados de sobrevivência materna, incluindo recorrência do tumor durante gravidez e sobrevida global (OS).

Se o relato do caso não especificou a idade gestacional exata, gravidez " precoce " e " tardia " foi definida como primeira e terceira trimestre, respectivamente. Nos casos em que a idade gestacional no parto foi descrita como "termo", a idade gestacional foi definida como 37 semanas. Pequeno para a idade gestacional (PIG) foi definido como menor que o $10^{\circ}$ percentil do peso ao nascer. O período de gestão expectante foi determinado pela duração entre o diagnóstico inicial de câncer de ovário durante a gravidez e a data do parto. 
OS era definido como o intervalo de tempo entre o diagnóstico de câncer de ovário e a data do óbito ou último acompanhamento. Se o relato de caso descreveu o tempo de sobrevivência desde a conclusão do tratamento inicial do câncer, este foi devidamente recalculado a partir do diagnóstico inicial de câncer de ovário. Eventos adversos graves foram definidos como: qualquer complicação significativa ao feto e/ou mãe, como ruptura ou torção do tumor, parto obstruído, metástase para unidade fetal-placentária, intra-útero fetal morte (IUFD) após a cirurgia, morte materna e morte neonatal ou natimorto. O estágio do câncer de ovário foi reclassificado adequadamente com base em Estadiamento da Federação Internacional de Ginecologia e Obstetrícia sistema (FIGO, 2009).

Casos tratados com quimioterapia foram agrupados como segue; combinação de platina e taxano, combinação de platina, antraciclina e ciclofosfamida ou outros. A normalidade das variáveis contínuas foi testada por Kolmo- teste GorovSmirnov, e descrito como média (ÆSD) ou mediana (intervalo) como apropriado. O teste exato de Fisher ou o teste do quiquadrado foi usado para comparação de variáveis categóricas conforme apropriado, descritas como probabilidades razão (OR) e intervalo de confiança de $95 \%$ (IC).

Para análise de sobrevivência, Log- teste de classificação foi usado para determinar a significância estatística para análise univariada. O teste de regressão de risco proporcional de Cox foi mais usado para identificar o preditor independente de sobrevida global na análise multivariada expressa como razão de risco (HR) com IC 95\%. As curvas de sobrevivência foram construídas pelo método Kaplan-Meier. P- valores inferiores a 0,05 foram considerados estatisticamente significativos (dois tailed).

\section{Resultados e Discussão}

Dos 3703 artigos identificados através da pesquisa de banco de dados, 102 artigos atenderam ao nosso critério de inclusão. Após avaliação adicional, 43 artigos foram removidos devido a uma descrição inadequada de resultado de sobrevivência materna, histologia obscura ou não divulgada, ou câncer primário não ovariano. Quatro artigos foram adicionados após revisar as referências dos artigos elegíveis. Finalmente, 11 artigos incluindo 105 casos foram elegíveis para a análise do estudo.

As características do paciente são idade média em o diagnóstico foi de 31 anos (variação, 19-44), e em 12,6\% dos casos, o paciente tinha 40 anos. Mais da metade dos casos eram nulíparas (52,9\%). O subtipo histológico mais comum foi seroso (47,6\%), seguido por mucinoso (27,6\%) e endometrioide (10,5\%). A maioria dos sintomas de apresentação comum foi dor abdominal ou pélvica $(26,7 \%)$ enquanto um terço foi detectado incidentalmente por meio do exame físico, ultrassom ou na hora da cesariana.

Os resultados da gravidez em quase metade dos casos foram diagnosticados durante o primeiro trimestre $(45,3 \%)$, e a maioria das gestações resultaram em nascidos vivos $(81,3 \%)$. O aborto eletivo era o motivo mais comum de gravidez inviável $(72,2 \%)$. Mais da metade dos casos $(57,7 \%)$ entregues a termo, e a maioria dos os nascimentos foram por cesariana $(71,6 \%)$.

O peso médio ao nascer foi de $2.725 \mathrm{~g}$ (variação, 600-4140) e 26,5\% dos neonatos eram classificados como SGA. Entre os casos que receberam quimioterapia in-utero py $(\mathrm{n}=21)$, não houve associação estatística com PIG (quimioterapia versus não quimioterapia, 19,0\% versus 12,9\%, OU 1,59, IC 95\% 0,35-7,21, p = 0,70).

Houve um caso de feto ventriculomegalia diagnosticada antes do início do útero quimioterapia com 20 semanas de gestação (Rouzi et al., 2009). No entanto, neonatal malformações relacionadas à quimioterapia intra-utero não foram relatado. Eventos adversos graves ocorreram em 21,9\% dos casos, com o mais comum sendo a ruptura do tumor durante a gravidez $(10,5 \%)$. Cinco $(6,4 \%)$ mortes neonatais foram descritas com o seguintes causas; três prematuridade, um de síndrome de aspiração de mecônio, sem descrição fornecida no caso restante (Chung et al., 1973; Day et al., 1958; Patsner et al., 1989; Sathanandan et al., 1977; Sayedur et al., 2002; Ueda et al., 1996). 
Um natimorto com 25 semanas de gestação foi atribuído à infecção intrauterina uma semana após a cirurgia. A mãe, neste caso, tinha estágio IV de adenocarcinoma mal diferenciado e morreu de sepse grave 2 semanas após o parto. O tumor tinha metástase para o sistema nervoso central e placenta (Patsner et al., 1989). No total, três (2,9\%) mortes maternas durante gravidez foram relatadas. Destes dois (ambos em estágio III), um morreu de infecção no íleo e disfunção respiratória 2 semanas após a cirurgia, e o outro morreu uma semana após a cirurgia devido a complicações pós operatórias (Betson et al., 1989; Sathanandan et al., 1977).

A gravidez foi tratada com expectativa em 59 casos $(56,2 \%)$ com uma duração média de 19 semanas (variação, 133). As proporções de gravidez em gestação foram $72,1 \%, 70,8 \%$, e $35,7 \%$, no primeiro, segundo e terceiro trimestres na época do diagnóstico de câncer, respectivamente $(\mathrm{p}<0,01)$. A duração mediana da gestação quando o câncer era diagnosticado no primeiro, segundo e terceiro trimestre foram 23,15 e 6 semanas, respectivamente $(p<0,01)$. A maioria das gravidezes resultou em nascimento, no entanto, dois casos resultaram em morte fetal devido a uma causa não especificada. Uma morte fetal intrauterina foi diagnosticada 4 semanas após anexectomia bilateral e ressecção sigmóide para adenocarcinoma mucinoso do ovário estágio III às 16 semanas de gravidez (Griffin et al., 1966).

O outro ocorreu 12 semanas após unilateral anexectomia para adenocarcinoma mucinoso ovariano estágio IA que foi realizado em 17 semanas. A maioria de os casos cirúrgicos foram realizados no segundo trimestre $(43,0 \%)$. Os tumores unilaterais representaram a maioria dos casos $(84,6 \%)$. A conservação fetal durante a cirurgia primária era relativamente comum $(61,9 \%)$. Na cirurgia primária durante a gravidez, anexectomia unilateral sozinha foi realizada mais comumente $(63,4 \%)$ do que anexectomia bilateral $(23,8 \%)$. Histerectomia concomitante ou a omentectomia no momento da cirurgia primária foi realizada em $16(15,8 \%)$ e $21(20,8 \%)$ dos casos, respectivamente. Linfadenectomia foi realizada em apenas um caso durante a gravidez (van Dessel et al., 1988).

A mediana o tamanho do tumor era de $12 \mathrm{~cm}$, sendo a maioria $10 \mathrm{~cm}$ ou maior $(72,3 \%)$. A idade gestacional no momento do diagnóstico do câncer não era associada ao tamanho do tumor $(\mathrm{p}=0,43)$ ou estágio $(\mathrm{p}=0,26)$. Estágio I os tumores foram mais comumente encontrados $(63,8 \%)$, seguidos pelo estágio III $(24,8 \%)$. Quase metade dos 105 casos foram tratados com quimioterapia $(55,2 \%)$, resultando na exposição de 21 fetos a drogas anticâncer no útero. Os regimes terapêuticos instaurados continham combinações de platina e taxanos, ou combinações de platina, antraciclina e ciclofosfamida; sem particular preferência por qualquer um dos regimes demonstrados na literatura (van Dessel et al., 1988; Griffin et al., 1966).

Um caso teve uma recorrência pélvica na $39^{a}$ semana de gravidez após submetidos a cistectomia unilateral em 6 semanas de gravidez para câncer em estágio IA. Houve $46(43,8 \%)$ gestações complicadas por epitélio câncer de ovário que a cirurgia citorredutora secundária adicional foi realizada após o período de gravidez. $\mathrm{O}$ detalhe do primário e as cirurgias secundárias desses 46 casos. A maioria $(56,5 \%)$ dos casos foi submetida a tratamento unilateral salpingo-ooforectomia na cirurgia primária seguida por salpingo-ooforectomia unilateral contralateral durante a cirurgia secundária (He et al., 2012).

Aproximadamente três quartos $(76,1 \%)$ resultaram em menopausa cirúrgica após a cirurgia secundária. Durante a cirurgia secundária, 80,4\% dos casos foram submetidos à histerectomia 19,6\%. A linfadenectomia foi realizada em quase metade dos casos $(45,7 \%)$ durante a cirurgia secundária. Cirurgia citorredutora ideal (tumor residual não maior que $1,0 \mathrm{~cm}$ no final da cirurgia) foi relatado em 97,3\% dos casos no tempo de cirurgia secundária sem doença bruta / residual sendo o estado citorredutivo mais comum $(94,6 \%)$ (He et al., 2012).

A idade gestacional no momento do diagnóstico, tipo de histologia, estágio, e eventos adversos graves foram significativamente relacionados a sobrevida materna em 2 anos por análise univariada (todos, p <0,05). Entre esses fatores, histologia indiferenciada e estágio IV doença foram associadas às piores taxas de sobrevivência de 2 anos (30,0\% e 25,0\%, respectivamente). Os fatores significativos determinados por análise univariada foram ainda avaliados por multivariada análise (Sayedur et al., 2002). 
Esta análise multivariada revelou que o estágio era o fator prognóstico independente mais forte para sobrevida e 2 anos a sobrevivência foi significativamente pior com a doença em estágio avançado (estágio III; HR 44,6, IC 95\% 5,73-347, p $<0,01$ e estágio IV; HR 399 (18,0-8812), p <0,01). Idade materna, cirurgia fetal conservadora, utero quimioterapia, tipo de quimioterapia, conduta expectante da gravidez e a via de parto não impactaram significativamente sobrevivência materna (todos, p> 0,05) (Ueda et al., 1996).

\section{Considerações Finais}

As principais conclusões da nossa revisão da literatura são que as mães os resultados da gravidez complicada por EOC são altamente contingentes no estágio do diagnóstico e tipo histológico. A maioria de gestações geridas com expectativa resultaram em sucesso neonatal resultados. Eventos adversos graves durante a gravidez foram menos frequentes do que foi observado em outros tipos histológicos de tumor ovariano. No entanto, mais de um quinto das gestações complicados por EOC estão associados a um evento adverso sério, que, em nosso estudo, foi associada à redução da sobrevida. Aproximadamente metade dos EOCs foram detectados no primeiro trimestre, forçando o paciente e o médico a tomar decisões difíceis sobre o curso de tratamento durante a gestação e se para continuar a gravidez.

A preservação da gravidez tem sido cada vez mais aceito em gestações complicadas por doenças malignas, e em nosso estudo, resultou em resultados gerais favoráveis para mãe e feto. Nem conduta expectante, fetal cirurgia de preservação ou administração de quimioterapia intra-uterina foram associados a mudanças nos resultados de sobrevida global, embora isso possa ser atribuído ao pequeno tamanho da amostra.

Como acontece com qualquer gravidez complicada por malignidade, o momento de parto deve ser equilibrado para levar em conta a maturidade fetal e bem-estar materno. Embora a taxa de parto prematuro é de aproximadamente $5 \%$ na população em geral, quase metade de gravidezes coincidentes com uma doença maligna darão parto prematuro, com o qual nosso estudo foi consistente $(42,3 \%)$. A maioria das admissões na unidade de terapia intensiva neonatal durante a gravidez câncer associado é devido à prematuridade. Como a evidência é acumular que nascimentos prematuros e mesmo prematuros são associados ao aumento da morbidade e mortalidade neonatal, é importante considerar opções alternativas, como iniciar tratamento durante a gestação e atrasando o parto até o termo.

$\mathrm{O}$ tratamento para EOC consiste principalmente em uma combinação de cirurgia e quimioterapia. A cirurgia continua sendo o esteio de tratamento para EOC, e vários estudos demonstraram o segurança da laparotomia ou laparoscopia na gravidez. A cirurgia secundária pode ser realizada após o parto, se necessário. Nossa revisão sistemática da literatura sugeriu que as gravidezes complicadas por câncer epitelial de ovário foram associadas com alta taxa de mortalidade materna e neonatal.

\section{Referências}

Akhter, N., \& Habib, A. Successful pregnancy outcome with coexisting primary epithelial ovarian carcinoma. Mymensingh Med J: MMJ. 22(3):599-602 Antonelli, N. M., Dotters, D. J., Katz, V. L., \& Kuller, J. A. Cancer in pregnancy: a review of the literature Part I. Obstet Gynecol Surv 51:125-34. Antonelli, N. M., Dotters, D. J., Katz, V. L., \& Kuller, J. A. Cancer in pregnancy: a review of the literature Part II. Obstet Gynecol Surv 51:135-42. Betson Jr, J. R., \& Golden, M. L. Primary carcinoma of the ovary coexisting with pregnancy; report of three cases. Obstet Gynecol 12(5):589-95. Chung, A., \& Birnbaum, S. J. Ovarian cancer associated with pregnancy. Obstet Gynecol 41:211-4.

Current FIGO staging for cancer of the vagina, fallopian tube, ovary, and gestational trophoblastic neoplasia. Int J Gynaecol Obstet 105:3-4. Day, A., \& Murray, D. J. Malignant ovarian neoplasms complicating pregnancy; report of two cases. Can Med Assoc J 15(78):941-4 (12). Estrela, C. (2018). Metodologia Científica: Ciência, Ensino, Pesquisa. Editora Artes Médicas. 
Research, Society and Development, v. 10, n. 11, e150101118704, 2021

(CC BY 4.0) | ISSN 2525-3409 | DOI: http://dx.doi.org/10.33448/rsd-v10i11.18704

Griffin, W. T. Pseudomucinous cystadenocarcinoma of the ovary associated with pregnancy. Case report. Mo Med 63(4):286-7.

Grimm D, Woelber L, Trillsch F, Keller-v Amsberg G, \& Mahner S. Clinical management of epithelial ovarian cancer during pregnancy. Eur J Cancer 50:963-71.

He, S. Y., Shen, H. W., Xu, L., Li, X. L., \& Yao, S. Z. Successful management of mucinous ovarian cancer by conservative surgery in week 6 of pregnancy: case report and literature review. Arch Gynecol Obstet 286(4):989-93.

Irving, J. A., Carinelli, S., \& Prat, J. (2006). Uterine tumors resembling ovarian sex cord tumors are polyphenotypic neoplasms with true sex cord differentiation. Modern Pathology, 19(1), 17-24.

Kwon, Y. S., Mok, J. E., Lim, K. T., et al. Ovarian cancer during pregnancy: clinical and pregnancy outcome. J Korean Med Sci 25:230-4.

Marcus, C. S., Maxwell, G. L., Darcy, K. M., Hamilton, C. A., \& McGuire, W. P. Current approaches and challenges in managing and monitoring treatment response in ovarian cancer. J Cancer 5:25-30.

McCluggage, W. G. (2011). Morphological subtypes of ovarian carcinoma: a review with emphasis on new developments and pathogenesis. Pathology, 43(5), 420-432.

Moran, B. J., Yano, H., Al Zahir, N., \& Farquharson, M. Conflicting priorities in surgical intervention for cancer in pregnancy. Lancet Oncol 8:536-44.

Morgan, R. J., Alvarez, R. D., Armstrong, D. K., Burger, R. A., Chen, L. M., Copeland, L., \& Hughes, M. (2013). Ovarian cancer, version 2.2013. Journal of the National Comprehensive Cancer Network, 11(10), 1199-1209.

Olson, S. H., Mignone, L., Nakraseive, C., Caputo, T. A., Barakat, R. R., \& Harlap, S. Symptoms of ovarian cancer. Obstet Gynecol;98,212-7.

Patsner, B., Mann Jr W. J., \& Chumas, J. Primary invasive ovarian adenocarcinoma with brain and placental metastases: a case report. Gynecol Oncol 33:1125.

Quirk, J. T., \& Natarajan, N. Ovarian cancer incidence in the United States, 1992-1999. Gynecol Oncol 97:519-23.

Rouzi, A. A., Sahly, N. N., Sahly, N. F., \& Alahwal, M. S. Cisplatinum and docetaxel for ovarian cancer in pregnancy. Arch Gynecol Obstet 280:823-5.

Sathanandan M, Visvanathan T, Sankaranarayanan K. Primary ovarian carcinoma in pregnancy. Ceylon Med J 1977;22(4 (Dec)):178-81.

Sayedur Rahman, M., Al-Sibai, M. H., Rahman, J., et al. Ovarian carcinoma associated with pregnancy. A review of 9 cases. Acta Obstet Gynecol Scand 81(3):260-4 (Review).

SEER Cancer Statistics Review, National Cancer Institute. Bethesda, MD, hhttp:// seer.cancer.gov/csr/1975_2010/i

Siegel, R., Ma, J., Zou, Z., \& Jemal, A. (2014). Cancer statistics, 2014. CA: a cancer journal for clinicians, 64(1), 9-29.

Smith, L. H., Dalrymple, J. L., Leiserowitz, G. S., Danielsen, B., \& Gilbert, W. M. Obstetrical deliveries associated with maternal malignancy in California, 1992 through 1997. Am J Obstet Gynecol 184:1504-12.

Stroup, D. F., Berlin, J. A., Morton, S. C., et al. Meta-analysis of observational studies in epidemiology: a proposal for reporting. Meta-analysis Of Observational Studies in Epidemiology (MOOSE) group. JAMA 283:2008-12.

Ueda, M., \& Ueki, M. Ovarian tumors associated with pregnancy. Int J Gynaecol Obstet 55:59-65.

van Dessel, T., Hameeteman, T M., \& Wagenaar, S S. Mucinous cystadenocarcinoma in pregnancy. Case report. Br J Obstet Gynaecol 95:527-9. 